\title{
Applications and challenges of plasma processes in NanoBiotechnology
}

\author{
F Rossi ${ }^{1}$ and P Colpo \\ European Commission, Joint Research Centre, Institute for Health and Consumer \\ Protection (IHCP), I-21020, Ispra (VA) Italy. \\ E-mail: francois.rossi@jrc.ec.europa.eu
}

\begin{abstract}
We present an overview of the possibilities offered by plasma technologies, in particular the combination plasma polymers deposition, colloidal lithography, ebeam lithography and microcontact printing, to produce micro and nanostructured surfaces with chemical and topographical contrast for applications in NanoBiotechnology. It is shown that chemical and topographical patterns can be obtained on different substrates, with dimensions down to few tenths of $10 \mathrm{~nm}$. The applications of these nanostructured surfaces in biology, biochemistry and biodetection are presented and the advantages and limitation of the plasma techniques in this context underlined.
\end{abstract}

\section{Introduction}

Since many years, the fabrication of surfaces presenting micron and submicron patterns with a welldefined geometry and chemistry has been the object of many research and publications in NanoBiotechnology $[1,2,3,4]$. For instance micro and nano-patterned surfaces with contrasted physico-chemical functionalities -e.g. protein adhesive/non-adhesive, hydrophilic/hydrophobic, acid/base...- provide unique tools in a large field of applications such as tissue engineering [5,6,7], cell behavior investigations $[4,5,8,9,10,11,12,13]$, or microfluidics $[14,15]$. Nanostructured surfaces have properties leading to very special effects related either to the kinetics of biochemical reactions at the interface $[16,17,18,19]$, or to their interaction with liquid (superhyhophobic or hydrophilic surfaces) $[20,21,22]$.

For a routine and cost-effective use of such micro-nanostructures, the fabrication method must rely on a well chosen combination of surface functionalisation and patterning techniques. Numerous methods have been developed for the production of such surfaces and are based essentially on the use of surface functionalisation techniques e.g. by Self Assembled Monolayers (SAMs) [23,24,25] or electrodeposition of functional polymers [26,27], combined with patterning techniques such as microcontact printing [28,29,31] or classical or advanced photolithography [30]. SAMs provide extremely well controlled surface chemistries but have several limitations particularly in relation to the substrate that has to be used - Gold or Silicon for thiol based or silane based chemistry respectively.

\footnotetext{
${ }^{1}$ To whom any correspondence should be addressed
} 
Besides, microcontact printing is the most widely used techniques for proteins and SAMs patterning but has some inherent limitation in terms of resolution due to stamp roof collapsing when high aspect ration structure are fabricated [31]. This is why other possibilities have been investigated, in particular those based on functional plasma polymers that can be deposited on virtually any substrate and at low temperatures. The plasma processes allow, by the choice of the right precursors, to deposit materials a very large range of properties and chemical functionalities, such as - $\mathrm{COOH}[32],-\mathrm{NH}_{2}[33,34]$ and their combination [35] or anti fouling polymers based for instance on polyethylene oxide (PEO) [36,37]. An excellent review of the different functionalities produced up to now can be found in [38].

Due to their variable degree of cross-linking linked to the discharge conditions, these coatings are resistant to classical polar solvents, making them compatible with almost all standard patterning techniques including lift-off processes $[39,40,41]$, but can also be selectively dissolved upon request. Plasma polymers can be then assembled in structures with sub-micron dimensions by combination of colloidal lithography $[39,44]$, deposition and etching, or as well as by direct modifications by electron beam lithography. Reactive etching is also a straighforward method to nanostructure polymer layers and then tune their wettability from superhydrophilicity to superhydrophobicity.

We develop here a summary of the work we performed, based on the use of plasma assisted deposition and plasma etching of functional polymers to produce patterns by a combination of techniques, namely colloidal and electron beam lithography and microcontact printing to address several issues in Nanobiotechnology: protein adsorption, high-sensitivity biodetection, control of stem cell maintenance and differentiation. We show thereby that plasma techniques provide a large range of interesting possibilities that offer many unique opportunities that cannot be easily obtained by other techniques, however at the expense of some degree of compromise for the control of surface chemistry. We provide general experimental details and present the combination of processes that have been developed for protein adsorption studies and cell cultures.

\section{Plasma based techniques.}

\subsection{Plasma polymerisation of functional films}

In the work summarized below, two different types of plasma polymers were used, namely plasma polymer of acrylic acid (pAA) containing $\mathrm{COOH}$ moieties and a protein and cell repellent plasma polymerized Polyethylene Oxide (PEO)-like surface. The choice of the acrylic acid is motivated by the fact that the carboxylic functions are able to promote a large amount of chemical reactions with different biomolecules (enzymes, antibodies, DNA...). However, other types of plasma polymers could be used as well (e.g. providing amine, epoxy moieties), to change the properties of the active layer and its interaction with adsorbed proteins (conformation, bioactivity, packing, denaturation etc...). The PEO-like polymer is resistant to adsorption of proteins in liquid solution but we will see that proteins are readily attached to the PEO-like surface in dry conditions, as the ones used in micro contact printing: this property offers a decisive advantages in terms of process simplicity and robustness $[42,43]$.

The deposition process of the plasma polymerized PEO-like and PAA films are described in details elsewhere $[32,44,45,39]$. Briefly, the systems used are capacitively coupled reactors with two symmetrical parallel-plate electrodes. For PEO-like coating, plasma polymerization is carried out by using a radio frequency generator $(13.56 \mathrm{MHz}$ ) in pulsed mode (time on $=10 \mathrm{~ms}$, time off $=100 \mathrm{~ms}$, nominal power $=5$ watts $)$ of pure Diethylene Glycol Dimethyl Ether (Di-glyme), $\left(\left(\mathrm{CH}_{3} \mathrm{OCH}_{2} \mathrm{CH}_{2}\right)_{2} \mathrm{O}\right)$ vapors (Sigma Aldrich, used as received). For PAA, acrylic acid vapors are mixed with Ar in pulsed $\mathrm{RF}$ mode (13.56 MHz; pulsed plasma: time on $=4 \mathrm{~ms}$; time off $=36 \mathrm{~ms}$ ). The films obtained are uniform and their thickness can be adjusted between 20 and $200 \mathrm{~nm}$. Characterisation of the PAA and PEO films can be found in $[32,36]$. Typically, the PAA films contain varying degree of carboxylic 
functions, depending on the power applied during the discharge. As discharge power is increased, the $\mathrm{O} / \mathrm{C}$ ration as measured by XPS decreases from $50 \%$ down to a few percent, increasing the stability of the films, which become insoluble in water for powers larger than $30 \mathrm{~W}$. For PEO films, similar results are obtained and the antifouling properties of the films are directly related to the importance of the CO-C peak in the C1S XPS spectrum, which decreases as the discharge power increases $[36,46]$. So the composition of the films, as well as the degree of cross-linking, vary continuously as the discharge power is increased, which make these films less well-defined as compared to SAMs for instance.

\subsection{Surface patterning using colloidal lithography}

The patterning of a surface by colloidal lithography consists in creating a 2-D crystalline structure of polystyrene (PS) beads on the PAA, those beads being used as masks during subsequent etching and deposition operation $[47,48]$. The mask self-assembly is performed by evaporation of solvent containing the PS beads in suspension. During the de-wetting and evaporation, the colloidal particles can be arranged in different geometrical configurations in order to minimize the free energy of the system $[39,44,45]$.

First, the PAA layer (typically thickness from 10 to $200 \mathrm{~nm}$ ) is deposited on a Si substrate as described above (figure 1a). Then, a micro-drop (Drop Volume $=5 \mu \mathrm{l}$ ) of PS beads (typically of 200 to $500 \mathrm{~nm}$ diameter) suspension is deposited on the $1 \mathrm{x} 1 \mathrm{~cm}^{2}$ samples surface. The bulk volume of the drop is removed by a micro-pipette, in order to obtain a very thin layer of PS beads suspension on the surface. The sample is then spun to produce a slow evaporation of the liquid. It is thus possible to create macroscopic homogeneous areas covered by mono-layered nano-beads of few $\mathrm{mm}^{2}$ with a surface coverage ranging typically of $90 \%$ (figure $1 \mathrm{~b}$ ). The nano-mask pattern is then transferred to the PAA layer by $\mathrm{O}_{2}$ plasma etching (Figure 1c). The etching operation has to be accurately controlled in order to create the nano-structures on the PAA layer and avoid over-etching. Once the polymeric nano-structures are created on the surface, the chemical contrast is formed by the plasma deposition of the PEO-like coating. The anti-fouling layer is deposited on the surface through the nano-masks (Figure 1d). The deposition is isotropic and the regions unprotected by the masks (both the partially and the totally uncovered regions) are coated.

The residual nano-sphere mask is then lifted-off by an ultrasonic treatment of the surfaces in ultra-pure water. The surface finally obtained is composed of areas of carboxylic functionalities of a few hundreds of nm surrounded by the anti-fouling layer (Figure 1e). The SEM and AFM images of Figure 2 show the typical 'nanodome' structure with an hexagonal distribution, the dimensions of which (typically a few hundreds of $\mathrm{nm}$ in diameter and few tenth of $\mathrm{nm}$ in height) depend on the initial size of the beads (here 500nm), the PAA layer thickness and the etching time. In particular, the distance between active areas can be adjusted by the duration of etching, which reduces the dimension of the mask and leaves the PAA domains farther apart. Structures of $200 \mathrm{~nm}$ up to $1000 \mathrm{~nm}$ and more can be obtained with this process, with coverage typically of $90 \%$ on a few $\mathrm{mm}^{2}$. Details of the experiments are given in $[67,68]$.

The shape, aspect ratio of the nanostructures can be controlled as well by tuning the plasma parameters [56].

For instance, reducing the working pressure leads to high aspect ratio structure thanks to the anisotropic character of the plasma etching [56]. Numerical simulations have been performed to predict the nanostructure shape as a function of plasma parameters [49]. This study shows that parameters such as substrate bias, electron temperature, and plasma density influence the ratio of ion flux hitting the nanoparticle interstice and the nanoparticles themselves. Fine-tuning of these plasma parameters can lead to a very accurate control of this plasma aided nanofabrication process. Pulsed bias can as well be used to finely tune the shapes of nanostructures in template-assisted nanoarray fabrication [50]. 


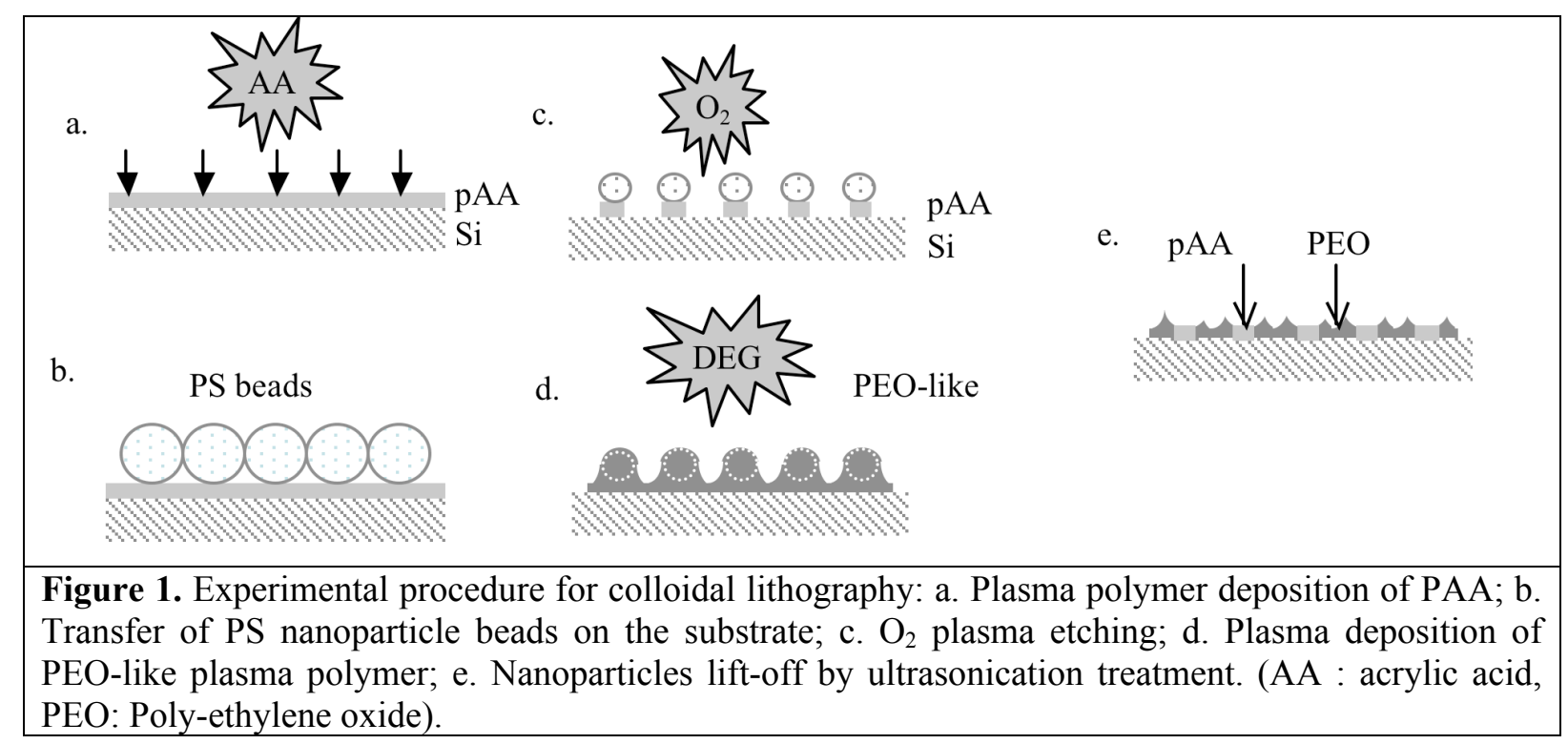

\subsection{Direct surface patterning using electron-beam lithography}

However, colloidal lithography is limited in the geometry of the patterns that can be produced (shape of the patterns, pitch, size, and distribution). This is why more conventional lithography techniques offer a large advantage, at the expense of time and cost. It has been shown that plasma polymers can be patterned by direct photolithography [51] but also with conventional processes using photoresists [36]. Nanopatterned surfaces can be as well fabricated by E-beam lithography using PMMA as resist $[52,53,54]$. Nanopatterns of typically $100 \mathrm{~nm}$ can be obtained by this approach. Lower dimensions can be obtained by optimizing the sequence of developing. E-beam lithography using PMMA as resist has been combined with plasma polymers enabling chemically nano contrasted surface fabrication [55]. In our work, we used the possibility of cross-linking water soluble PAA directly by the electron beam. The process of fabrication is described in Figure 3. First, a $20 \mathrm{~nm}$ layer of PEO-like polymer is plasma deposited on a Si substrate in order to form the non-adhesive matrix [28]. Then a second water-soluble layer is deposited by plasma polymerization of acrylic acid in the monomer rich region. The conditions of deposition of this layer are similar to the one of PAA previously described but with a lower power of deposition (nominal power of 10 watts, operating in pulsed mode with a $t_{\text {on }}$ of $4 \mathrm{~ms}$ and a duty cycle of $10 \%$ ). The film thus obtained is not cross-linked and dissolves readily in water.

After deposition of the water-soluble layer, the surface is exposed to the electron-beam with an energy of $20 \mathrm{keV}$ and incident dose of $1600 \mathrm{mC} / \mathrm{cm}^{2}$, then immersed for 5 minutes in water and dried under nitrogen flow. Local cross-linking of the polymer is thus used to produce the stable domains of PAA. The chemistry of the stabilized PAA layer has been determined by $\mu$-spot XPS on a micro patterned surface $\left(100 \times 100 \mathrm{~mm}^{2}\right)$. XPS analysis reveals that the chemistry of the stabilized layer is modified as compared to the initial film, because of the cross-linking occurring during electron beam irradiation. The chemistry of the film thus produced is similar to plasma polymerized acrylic acid layer produced at optimum plasma density and used for bio specific applications with a percentage of retention of $\mathrm{COOH}$ groups around $7-10 \%[56]$. 

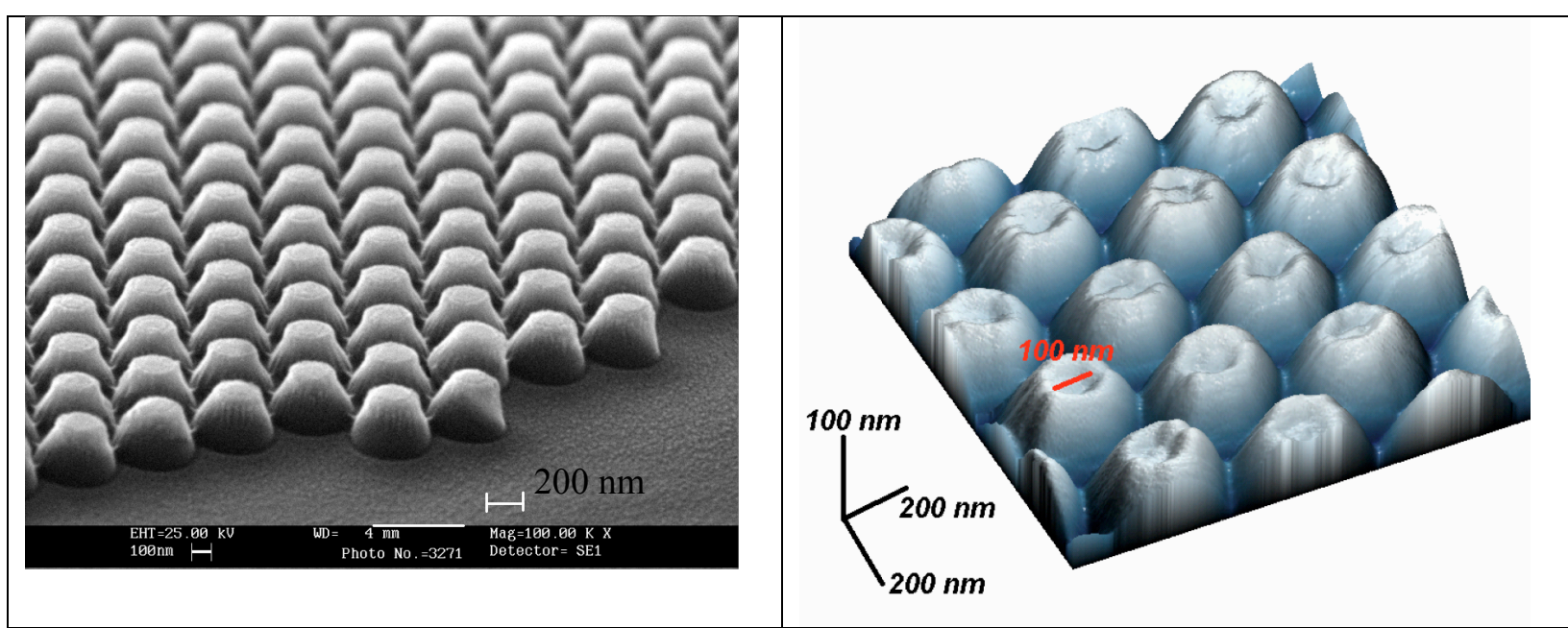

Figure 2. SEM and AFM pictures of the structures obtained by colloidal lithography. Reproduced with permission [57]

Figure 4 shows AFM and SEM images of functional nano-structures with different heights and shapes created with the patterning process described in this study. Well-defined features sizes down to 200 $\mathrm{nm}$ in lateral resolution can be fabricated with several heights (from $80 \mathrm{~nm}$ to $500 \mathrm{~nm}$ ). It must be noted that the resolution limit obtained (or the order of $100 \mathrm{~nm}$ ) depends on the e-beam energy and can be much improved by working at lower energies (typically down to $5 \mathrm{keV}$ ) provided by a dedicated instrument. These images proves the feasibility of combining electron-beam lithography and plasma processes for direct patterning of surfaces with well defined nano-scale dimensions. More experimental details can be found in [69].

\begin{tabular}{|l|l|}
\hline substrate & $\begin{array}{l}\text { Figure 3. Electron-Beam lithography on } \\
\text { plasma polymers: A PEO layer is first } \\
\text { deposited on a Si substrate (a). A second } \\
\text { layer of unstable acrylic acid plasma } \\
\text { polymer is deposited on the PEO (b). e- } \\
\text { beam irradiation stabilizes locally the } \\
\text { PAA (c). After washing, one obtains a } \\
\text { nanostructure of stable PAA domains on } \\
\text { a non-adhesive PEO background (d). }\end{array}$ \\
c. &
\end{tabular}



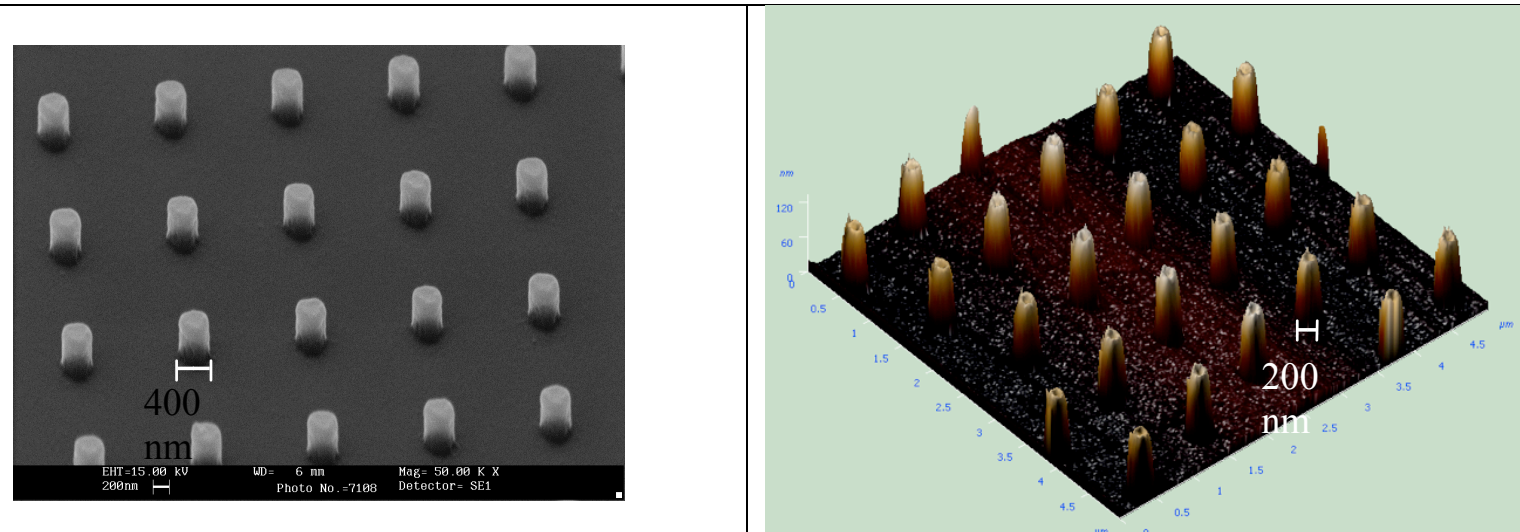

Figure 4. SEM (left) and AFM (right) pictures of nanostructures obtained by e-beam patterning of an unstable PAA film. Typical dimensions in height can vary from 100 to $500 \mathrm{~nm}$.

\subsection{Plasma polymer PEO-like films as platform for proteins and cell patterning.}

Protein and cell patterning plays an important role for biological and biochemical applications [30]. Challenges reside in developing of surfaces that can support for long time period well-defined biomolecule patterns in a non-adhesive matrix. A difficulty is that protein adsorption at the interface, which results from a complex interplay of electrostatic, hydrophobic and chemical forces [58] renders the results hard to control. An improvement in the spatial distribution of protein adsorption onto surfaces can be obtained by using microcontact printing. The advantage of the approach is the transfer directly from the stamp to the surface the chemicals, ligands or proteins of interest $[59,60,61]$. This technique has previously been successfully applied in producing highly contrasted chemical functionalities with sharp contours [62] and to pattern proteins for the study of bio/non-bio interactions and for cell attachment $[43,63,64,65]$. The main difficulties to design these surfaces concerns le anti fouling properties of the background to keep the proteins or cell microarrays stable in time. In our work, we have optimised a platform based on plasma polymerized PEO like-films for the patterning of human umbilical cord blood - derived neural stem cells. The goal is to study cellular maintenance and differentiation when interacting with polypeptide micropatterns on a biologically non-adhesive surface [49-50]. To produce these surfaces, microcontact printing has been used as a mean to deposit controlled, geometric patterns of Poly-L-lysine (PLL) and fibronectin (FN) on glass substrates previously coated with a protein resistant, cell repellent plasma polymerized PEO film. The PEO layer has the important characteristic of being cell repellent in solution but protein adhesive under the dry conditions used during the microcontact printing operation $[42,66]$. The composition of the PLL or FN inking solution and the $\mathrm{pH}$ were critical to the quality of the pattern, which under optimised conditions could be effectively transferred to the substrate where it was stable for more than four weeks $[43,65]$. Details of the experiments are reported in [43].

Well-defined microstructures of PLL over a protein free background have been fabricated, as can be seen in figure 5, which shows fluorescence microscopy (Figure 5 A-C) and Time of Flight Secondary Ion Mass Spectroscopy (ToF-SIMS) images (Figure 5 D-G) of the polypeptide pattern. The geometry of the PLL pattern has been chosen to suit the desired cell response required for the study. Long inter-patch distances (squares $165 \mu \mathrm{m}$, spaced $400 \mu \mathrm{m}$ ) are preferred for cell migration studies, while short distances (squares $100 \mu \mathrm{m}$, spaced $100 \mu \mathrm{m}$ ) are better for cell differentiation and outgrowth projection studies. The extension of cell protrusions was also monitored on interconnected islands by bridging the squares with $15 \mu \mathrm{m}$ width lines (Figure 5C). The PLL ToF-SIMS positive imaging, ascribed to $\mathrm{C}-\mathrm{N}$ bonding (Figure 5D, 5F) clearly shows a large difference in intensity of this ion between the printed area and surrounding PEO background. In comparison, the images derived from peaks associated with $\mathrm{PEO}$, given by $\mathrm{C}_{3}-\mathrm{H}_{3}-\mathrm{O}$ bond mapping (Figure 5E, 5G), show a much less 
distinct contrast between the printed areas and the surrounding background PEO. From these results it can be concluded that the PLL is only delivered in the squares while the surrounding background is totally PLL free and that the PLL layer is sufficiently thin or has incomplete coverage (at a sub-micron level) that a significant contribution to the PEO ion image comes from the underlying layer. Fluorescence imaging of the samples after immersion in water for one month revealed a good stability of the PLL attachment to the PEO substrate.

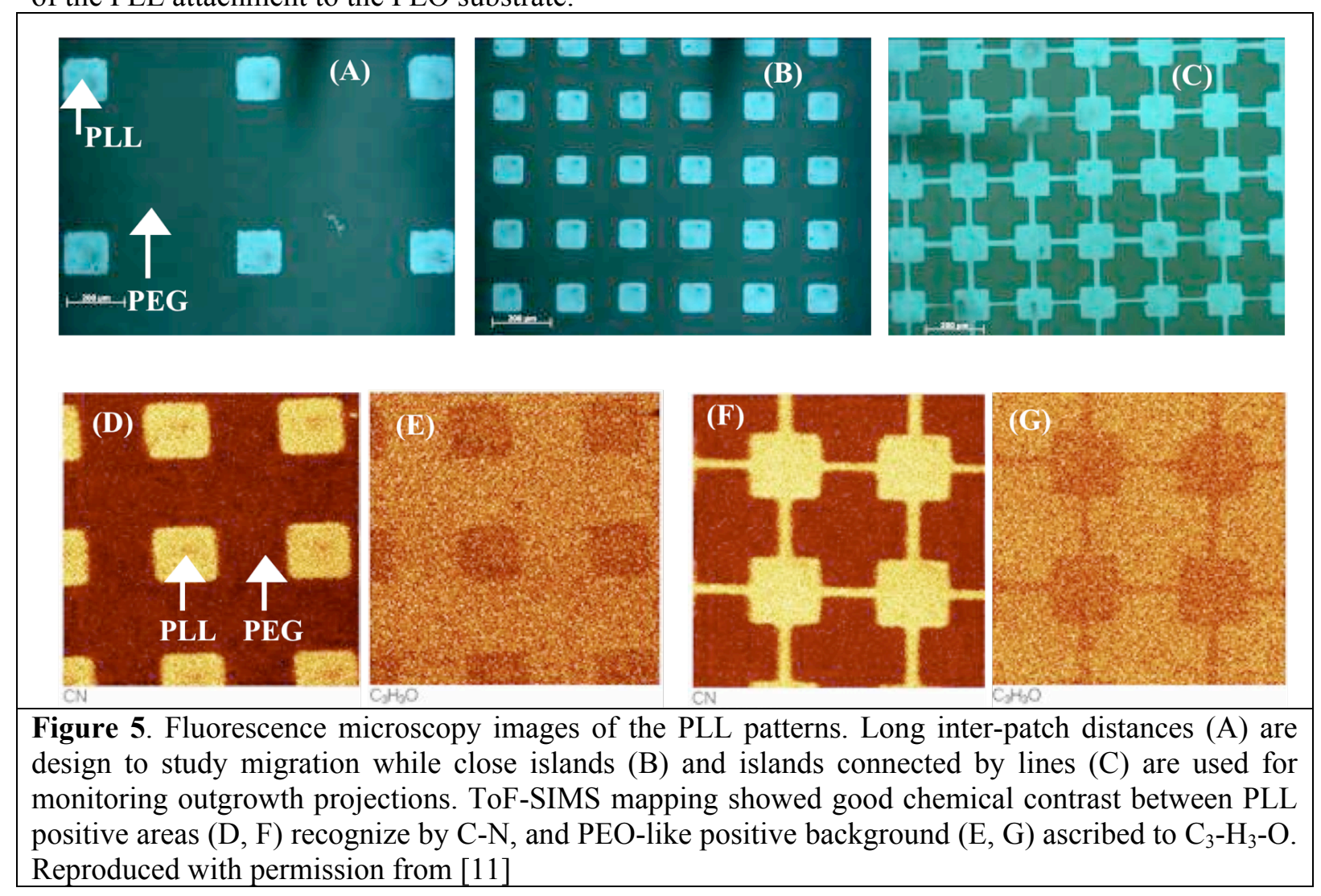

\section{Application to bio interfaces}

\subsection{Protein adsorption on nanopatterns}

The nanostructures produced by colloidal and electron-beam lithography have been extensively studied with different biological tests and protein adsorption experiments $[17,67,68,69,70,73]$. We present below two significant examples indicating the particular behaviour of nanostructures as compared to non-patterned surfaces.

In order to illustrate that the potential of these surfaces as biosensors platforms, nanostructured surfaces produced by e-beam stabilization of acrylic acid on a PEO background have been exposed to a fluorescent Poly-L-Lysine (PLL) solution. The fluorescent contrast observed in figure 6 after the incubation shows that PLL is adsorbed selectively on the PAA domains and that the PEO-like has kept its anti adhesive properties. These results show that plasma polymers combined with E-Beam lithography are a very promising technique to produce nanostructured surfaces with chemical nano contrast for protein nanopatterning in sensing and biology applications.

Quartz crystal microbalance with dissipation (QCM-D) (Qsense, Sweden) has been used for quantitatively compare the mass of proteins absorbed on uniformly functionalized and nano-structured surfaces fabricated by colloidal lithography. The mass of proteins absorbed on the crystals was 
estimated by the Sauerbrey equation, corrected for the water coupled to the proteins. For the determination of variation of frequency (i.e. mass absorption) due to protein adsoption, $25 \%$ of the measured mass, considered as the contribution of the water in the measurement was subtracted: this estimate is consistent with the typical average retention of water in proteins [22]. The number of absorbed molecules was then calculated by dividing the estimated mass by the molecular weight of the molecule and by multiplying it by the Avogadro's number.

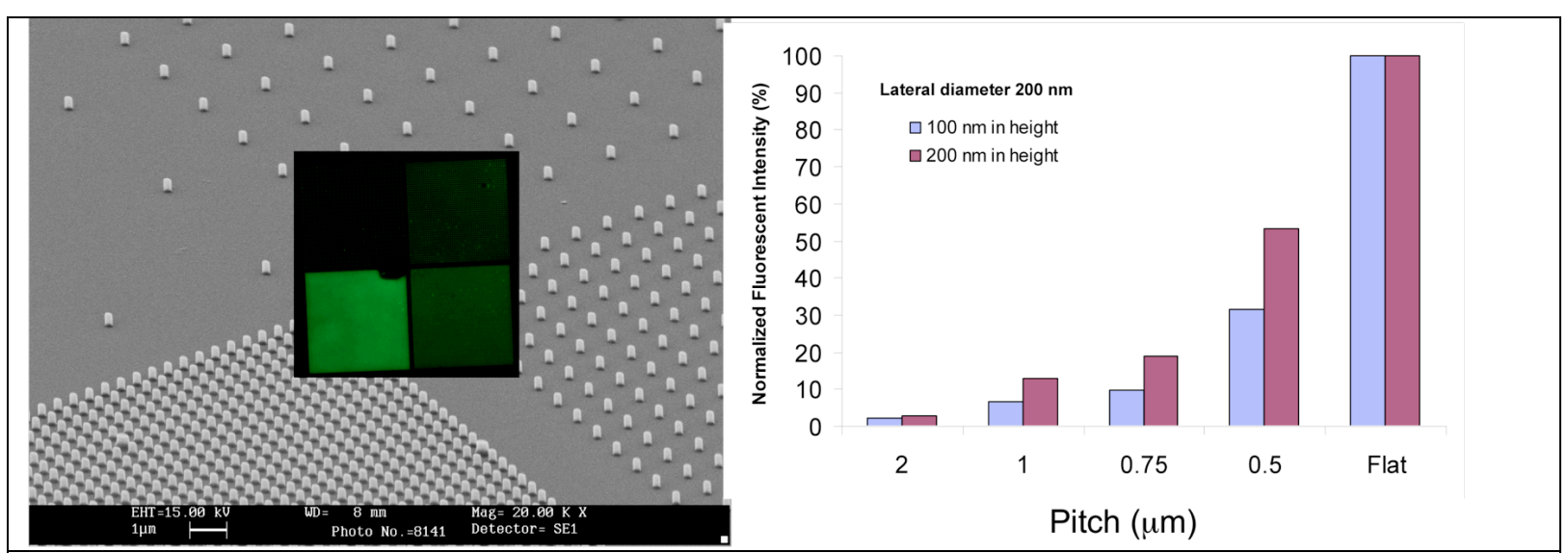

Figure 6. (a): SEM and fluorescent (included inside the SEM image) of nanopillars $(\mathrm{h}=100 \mathrm{~nm}, \mathrm{~d}=$ $200 \mathrm{~nm}$ ) of stabilized PAA on PEO-like surface and corresponding relative fluorescent intensity after incubation with PLL for different height and pitch of pillars. Reproduced with permission from [69].

Both the probe (human $\mathrm{IgG}$ ) immobilisation and the antibody/antigen (human IgG/Anti-human IgG) recognition have been monitored on-line. The nano-patterned quartz resonator was held in the measurement cell and the baseline was recorded when the carrier buffer (PBS: $10 \mathrm{mM} \mathrm{PBS,} \mathrm{pH} \mathrm{7.4)}$ was flowing in the reaction cell. For the probe immobilisation, Human IgGs with concentrations ranging from 1 to $15 \mu \mathrm{g} / \mathrm{ml}$ in PBS solution were injected in the liquid cell. The results in Figure 7 are expressed as the difference between the values measured after the rinsing step and before the sample injection. The same buffer solution was used for all the experiments. In these conditions, the variation is related only to compounds fixed on the surface during the reaction [71,72].

In the case of nano-structures, the areas covered by PEO (anti fouling matrix between the nanostructures and the areas non-structured areas) are considered totally protein repellent since the measurement by QCM did not show detectable protein adsorption on PEO layers (data not shown).

Figure 7 shows the result of the immuno-reaction as a function of primary antibody concentration. It can be seen that limit of detection is shifted toward lower concentration, with best results obtained at smaller nanopattern dimensions (200 nm nanostructure). Similar enhancement has been observed with different techniques (SPRi, QCM, ELISA test) $[67,68,73,74]$. The level of amplification depends on the size of the nanostructure and increases as the size of the patterns decreases. A possible explanation is linked to a better orientation of the antibodies on a nanostructured surfaces and a reduction of the steric hindrance linked to the antibodies adsorption on the edges of the structures, making binding reactions more efficient on the nanostructures as compared to uniform surfaces [75]. 

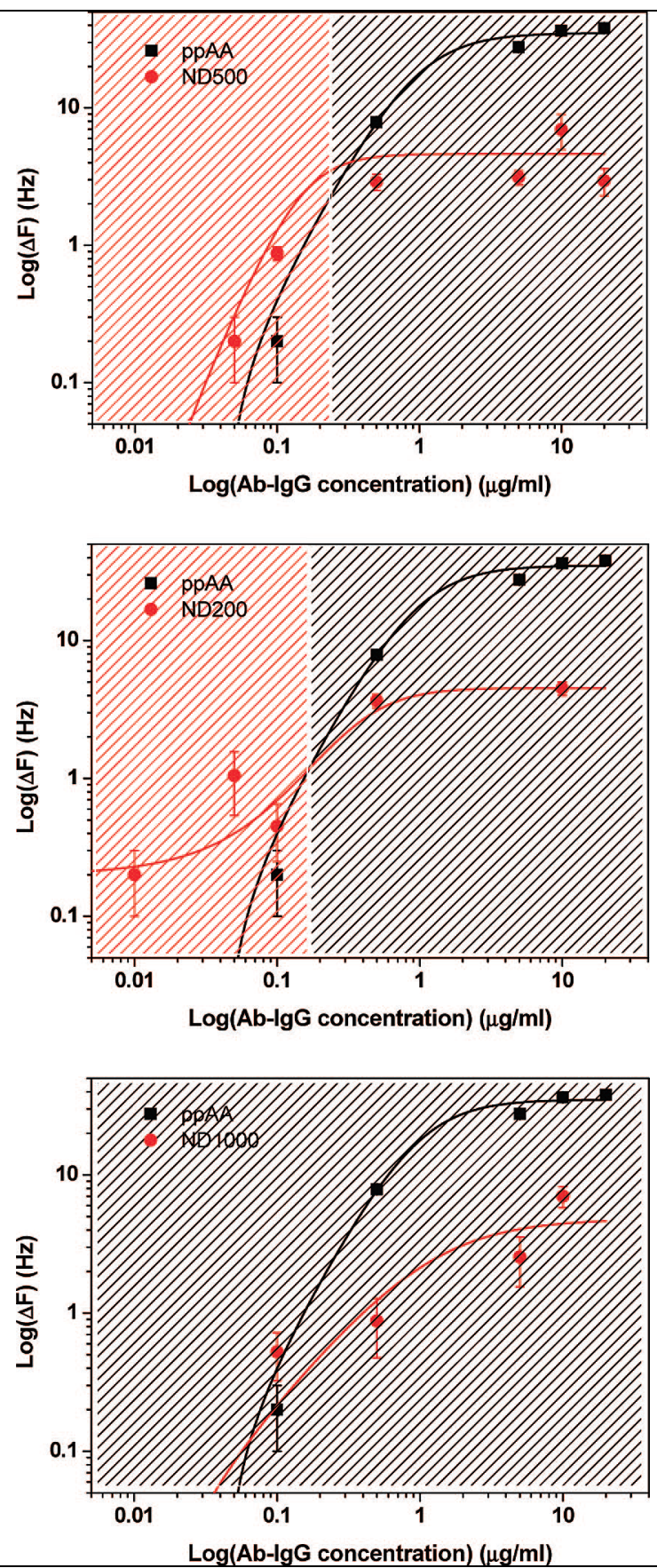

Figure 7. Dose-response curves for the flat ppAA and the nanostructured surfaces. The solid lines are the sigmoidal-shaped functions best-fitting the calibration curves. The curve for ppAA is reported in each graph for direct comparison with the nanostructured surfaces. Reproduced with permission [71]

\subsection{Cell culture experiments}

Fibronectin and PLL microcontact printed surfaces were used to study the patterned growth of neural stem cells and as a model to study different processes regarding stem cell neural lineage commitment. Human umbilical cord blood derived neural stem cells (HUCB-NSC) [76] have been incubated on the polypeptide functionalized surfaces. Previous studies in non-patterned surfaces [77] showed that the density of plating and the type of polypeptide used for attachment can change the HUCB-NSC 
commitment to a specific lineage (neuronal, astrocytic, olegodendroglial). In this work, the cells attached to the FN and PLL domains grow following the pattern but show a behaviour that is sensitive to both the geometry and the cell density. It has been reported that different cell morphologies can influence stem cell commitment [78]. In this study, it was noticed that cells on densely crowded islands stayed in non-differentiated mode longer than low populated samples where cell commitment to a differentiated mode is favoured. By varying the conditions of culture (with and without serum, with and without growth factor, on FN and PLL patches), we found that the differentiation of HUCBNSC could be modulated to a large extent, the population of neurons, astrocytes and oligodendrocytes varying between $10 \%$ and $80 \%$ depending on the conditions (see Figure 9) $[11,76,77]$.

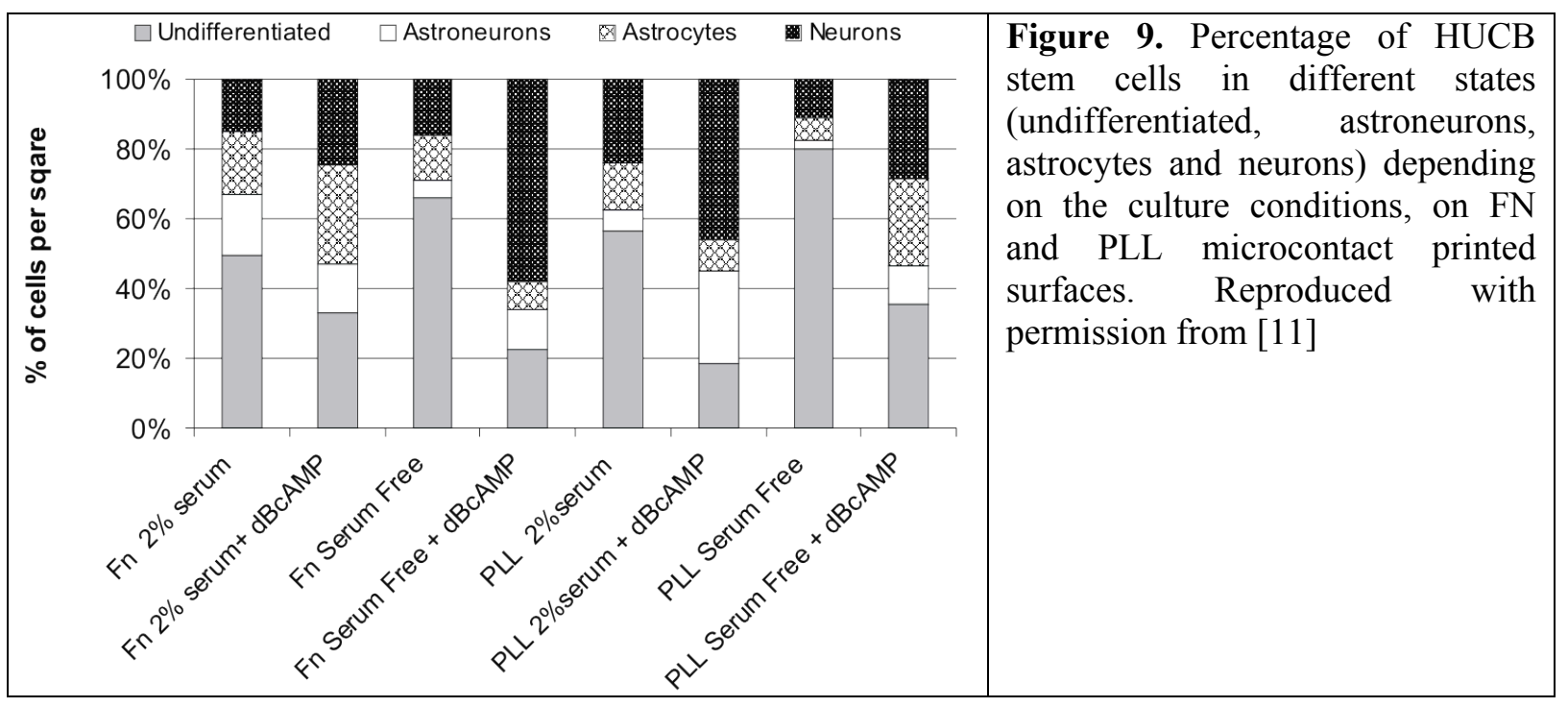

\section{Conclusions and perspectives}

There is a constant need of new technologies for research innovation in healthcare, environment monitoring and toxicology studies. For these applications, there is a recurrent requirement for the development of new devices that can be used to detect disease marker, pollutant or to improve the reliability of in vitro testing. Bio/non bio interface is the common cornerstone for the fabrication of such a device. Proteins and cells must be immobilised on surfaces in a manner that all their functionality are kept intact. In this domain, surface functionalisation and patterning plays a pivotal role. Many articles have been published on this topics using a variety of different methodologies.

Nevertheless, the proposed techniques are often difficult to implement routinely and suffer from reproducibility issues. Most of these methods are based on non-trivial wet chemistry (SAMs, hydrogels...). This paper shows that plasma technology represents an interesting alternative. Derived from plasma processes using in semi conductor industry, the processes of deposition or etching operation quality can be highly controlled by mass or optical spectrometry and process fully automated.

In this article, we have shown that plasma technologies, and in particular deposition and etching can be combined with colloidal and electron-beam lithography as well as microcontact printing for production of high quality micrometric and sub-micrometric patterned surfaces with arbitrary geometries and controlled chemistry. The resolutions that can be obtained vary with the techniques used from the micrometer scale down to a few tens of $\mathrm{nm}$. We have seen that the major advantage of plasma polymers is related to the large range of properties that can be obtained, and their relative stability with time and against different solvents. Moreover, due to the versatility of the plasma processes, these techniques can be easily extended to a large panel of surface chemistries without constraints in terms of substrates, and many studies are currently carried out on the use of alternative 
chemistries, which could benefit from the same approach and extend its application. This is however done at the expense of control of chemistry of the surface, the plasma polymers chemical properties being intrinsically more ill defined as compared to SAMs for instance. We also feel that the combination of conventional biochemistry/chemistry and plasma processing techniques opens a large range of possibilities and offer very interesting opportunities for cell cultures as well as bioanalytics. The approach can also be extended by using for instance other combinations of techniques (ion beam, UV treatments) or stimuli responsive materials $[79,80]$ for e.g. modulating the reactivity of the films and developing new structures. A major challenge of these processes will be optimization of the robustness and control of surface properties of the platforms obtained to make them applicable at a large scale at low cost.

Plasma technology has all the necessary requisites to penetrate successfully all life sciences related applications.

\section{References}

[1] Niemeyer CM, Mirkin C (eds.), Nanobiotechnology Concepts, Applications and Perspectives, 2004 Wiley-VCH Verlag GmbH

[2] Walter G, Bussow K, Lueking A and Gloker J, 2002 Trends. Mol. Med. 8, 250

[3] Zhu B, Zhang Q, Lu Q, Xu, Y, Yin J, Hu J and Wang Z, 2004 Biomaterials, 25, 4215

[4] Blawas A S and Reichert W M, 1998 Biomaterials, 19, 595

[5] Khademhosseini A, Borenstein J, Takayama S, Toner M, Micro and Nanoengineering of the Cellular Microenvironment: Technologies and Applications" Artech House Publishing, 627p. USA (2008)

[6] Curtis A and Riehle M 2001, Phys. Med. Biol. 46, R47

[7] Arnold M, Cavalcanti-Adam A, Glass R, Blummel J, Eck W, Kantlehner M, Kessler H and Spatz J P 2004, ChemPhysChem. 5, 383

[8] Thomas C H, Lhoest J B, Castner D G, McFarland C D and Heal K E, 1999 Trans ASME J. Biomech. Eng. 121 (1), 40

[9] Kam L, Shain W, Turner J N and Bizios R, 1999 Biomaterials, 20, 2343

[10] Gadegaard N, Martines E, Iehle M O, Seunarine K and Wilkinson C D W, 2006 Microelectronics Engineering, 83, 1577

[11] Bużańska L, Ruiz A, Zychowicz M, Rauscher H, Ceriotti L, Rossi F, Colpo P, Domańska-Janik K and Coecke S. 2009 Acta Neurobiol. Exp., 69 24-36

[12] Fink J, Théry M, Azioune A, Dupont R, Chatelain F, Bornensa M and Piel M, 2007 Lab Chip, 7, 672680

[13] Dalby M J, Berry C C, Riehle M O, Sutherland D S, Agheli H and Curtis A S G, 2004, Experimental Cell Research 295, 387-394

[14] Whitesides G M, 2006 Nature 442, 368

[15] Seemann R, Brinkmann M, Kramer E J, Lange F L, and Lipowsky R, 2005, PNAS 102 (6) 1848-1852

[16] Beni V, Valsesia A, Colpo P, Brétagnol F, Rossi F and Arrigan D W M 2007 Electrochemistry Communications, 9, 1833

[17] Valsesia A, Colpo P, Meziani T, Lisboa P, Lejeune M, and Rossi F, 2006 Langmuir, 22 (4), 1763

[18] Krishnamoorthy S. and Himmelhaus M. 2008 Adv. Mat., (20), 2782-2788

[19] Nathaniel L. Rosi and Chad A. Mirkin Chem. Rev. 2005, 105, 1547-1562

[20] Lacroix LM, Lejeune M, Ceriotti L, Kormunda M, Meziani T, Colpo P, Rossi F, Surface Science 592 (2005) $182-188$

[21] Tsougeni K, Vourdas N, Tserepi A, and Gogolides E, Langmuir 2009, 25(19), 11748-11759

[22] Han Z, Tay B, Tan C, Shakerzadeh M, Ostrikov K, ACS Nano, 2009, 3 (10), 3031-3036

[23] Love JC , Estroff LA , Kriebel JK , Nuzzo RG , Whitesides GM (2005). Chem Rev 105 : 1103 - 1169

[24] Lercel M J, Craighead H G, Parokh A N, Seshadri K and Allara D L, 1996 Appl. Phys. Lett. 68, 1504

[25] Lussi J W, Tang C, Kuenzi P A, Staufer U, Csucs G, Voros J, Danuser G, Hubbell J A and Textor M, 2005 Nanotechnology, 16, 1781

[26] Haddour H, Cosnier S, and Gondran C, 2005 Journal of American Chemical Society, 127, 5752-5753

[27] Ramanavičius A, Ramanavičiene A and Malinauskas A 2006 Electrochim. Acta. 51 6025-6037

[28] Renault J P, Bernard A, Bietsch A, Michel B, Bosshard H R, Delamarche E, Kreiter M, Hecht B and Wild U P J, 2003 Phys. Chem. B. 107, 703 
[29] Taha H, Marks R S, Gheber L A, Rousso I, Newman J, Sukenik C and Lewis A, Appl. Phys. Lett. 83, 1041 (2003)

[30] Falconnet D, Csucs G, Grandin HM, Textor M (2006). Biomaterials 27 : 3044 - 3063

[31] Delamarche E, Schmid H, Michel B and Biebuyck H, 1997 Advanced Materials, b, 741.

[32] Rossini P, Colpo P, Ceccone G, Jandt K D and Rossi F, 2002, Materials Science and Engineering C, 1050, 1

[33] Manso M, Valsesia A, Ceccone G, Colpo P and Rossi F, 2004 Appl. Nanoscience, 1(1), 47

[34] Lejeune M, Brétagnol F, Ceccone G, Colpo P and Rossi F, 2006 Surface and Coatings Technology, 200, 5902

[35] Favia P, De Vietro N, Di Mundo R, Fracassi F and d'Agostino R, 2006 Plasma Process. Polym., 3, 6674

[36] Brétagnol F, Lejeune M, Papadopoulou A, Hasiwa H, Rauscher H, Ceccone G, Colpo P and Rossi F, 2006 Acta Biomaterialia, 2 (2), 165

[37] Chu L, Knoll W and Förch R, 2006 Plasma Process. Polym. 3, 498-505

[38] Shyong Siow K, Britcher L, Kumar S, and Griesser H J 2006, Plasma Process. Polym. 3, 392-418

[39] Valsesia A, Colpo P, Manso Silvan M, Meziani T, Ceccone G and Rossi F, 2004 Nano Lett. 4(6); 1047

[40] Baralia G G, Pallandre A, Nysten B and Jonas A M, 2006 Nanotechnology, 17, 1160

[41] Sardella E, Favia P, Gristina R, Nardulli M and d'Agostino R, 2006 Plasma Process. Polym. 3 456469

[42] Pan V, McDevitt TC, Kim TK, Leach-Scampavia D , Stayton PS, Denton DD , Ratner BD (2002) Plasma Polym 7, 171 - 183

[43] Ruiz A, Buzanska L, Ceriotti L, Brétagnol F, Coecke S, Colpo P and Rossi F 2008 J. Biomater. Sci. Polymer Edn., 19(12):1649-57

[44] Lopez L C, Gristina R, Ceccone G, Rossi F, Favia P and d'Agostino R. 2005 Surface \& Coatings Technology 200 1000- 1004

[45] Valsesia A, Manso Silvan M, Ceccone G, Gilliland D, Colpo P and Rossi F 2005 Plasma Process. and Polym., 2, 334-339

[46] Sardella E, Gristina R, Senesi G S, d'Agostino R and Favia P 2004 Plasma Process. Polym. 1, 63-72

[47] Agheli H and Sutherland D S, 2006, IEEE Trans. on Nanobioscience 5 (1) 9-15

[48] Michel R, Reviakine I, Sutherland D, Fokas C, Csucs G, Danuser G, Spencer N D and Textor M, 2002, Langmuir 18, 8580-8586

[49] Yuan L, Zhong X and Ostrikov K. Nanotechnology 19 (2008) 155304 (10pp)

[50] Wu X, Zhong X, Ostrikov K, App.Phys Lett. 92, 223104 (2008)

[51] Dirani A, Wieder F, Roucoules V, Airoudj A and Sopera O, 2010, Plasma Process. and Polym. 8, 571581

[52] Chen W and Ahmed H, 1993 Appl. Phys. Lett. 62, 1499

[53] Vieu C, Carcenac F, Pepin A, Chen Y, Mejias M, Lebib A, Manin-Ferlazzo L, Couraud L and Launois H, 2000 Appl. Surf. Sci. 164, 111

[54] Baralia G G, Pallandre A, Nysten B and Jonas A M, 2006 Nanotechnology, 17, 1160

[55] Bretagnol F,Ceriotti L, Valsesia A, Sasaki T, Ceccone G, Gilliland D, Colpo P, and Rossi F, Nanotechnology 18 (2007) 135303 (5pp)

[56] Detomaso L, Gristina R, Senesi G S, d'Agostino R and Favia P, 2005 Biomaterials, 26 (18), 3831

[57] Rossi F, Bretagnol F, Valsesia A and Colpo P, 2008 Eur. Phys. J. Appl. Phys. 43, 277-281

[58] Latour R., 2006 Journal of Biomedical Materials Research Part A Volume 78A, 4, 843-854

[59] Ruiz S A and Chen C S 2007 Soft Matter, 3, 1-11

[60] Kumar A and Whitesides G M 1993 Appl. Phys. Lett., 63, 2002-4

[61] Xia Y and Whitesides G M, 1998 Angew. Chem. Int. Ed. 37, 550-75.

[62] Csucs G, Michel R, Lussi J W, Textor M and Danuser G 2003 Biomaterials, 24, 1713-20

[63] Chang J C, Brewer G J and Wheeler B C, 2003 Biomaterials, 24, 2863-70.

[64] Suh K Y,Seong J, Khademhosseini A, Laibinis P E and Langer R 2004 Biomaterials, 25, 557-563.

[65] Ruiz A, Valsesia A, Brétagnol F, Colpo P, and Rossi F 2007 Nanotechnology, 18, 531-536.

[66] Delamarche E, Donzel C, Kamounah F S, Wolf H, Geissler M, Stutz R, Schmidt-Winkel P, Michel B, Mathieu H J, and Schaumburg K, 2003 Langmuir, 19, 8749-8758.

[67] Valsesia A, Colpo P, Meziani T, Brétagnol F, Lejeune M, Rossi F, Bouma A and Garcia-Parajo M, 2006 Advanced Functional Materials, 16, 1246 
[68] Valsesia A, Meziani T, Brétagnol F, Colpo P, Ceccone G and Rossi F, 2007 J. Phys. D: Appl. Phys. 40, 2341

[69] Brétagnol F, Valsesia A, Sasaki T, Ceccone G, Colpo P and Rossi F 2007 Advanced Materials, 19 (15), 1947 (2007)

[70] Brétagnol F, Kylian O, Hasiwa M, Ceriotti L, Rauscher H, Ceccone G, Gilliland D, Colpo P and Rossi F, 2007 Sensors and Actuators B, 123, 283

[71] Valsesia A, Mannelli I, Colpo P, Bretagnol F and Rossi F, 2008, Anal. Chem. 80, 7336-7340

[72] Valsesia A, Colpo P, Mannelli I, Mornet S, Bretagnol F, Ceccone G and Rossi F 2008 Anal. Chem. 80, (5) $1418-1424$

[73] Lisboa P, Valsesia A, Colpo P, Rossi F and Mascini M, 2010 Analytical Letters, 43: 1556-1571

[74] Lisboa P, Valsesia A, Manelli I, Mornet S, Colpo P and Rossi F, 2008 Adv. Mater., 9999, 1-7

[75] Krishnamoorthy S. and Himmelhaus M. 2008 Adv. Mater., (20), 2782-2788.

[76] Buzanska L, Jurga M, Stachowiak E K, Stachowiak M K and Domanska-Janik K 2006, Stem Cells Dev. 15, 391-406.

[77] Buzanska L, Habich A, Jurga M, Sypecka J and Domanska-Janik K, 2005 Toxicology in Vitro, 19, 991999.

[78] McBeath R, Pirone D M, Nelson C M, Bhadriraju K and Chen C S, 2004 Develop. Cell 6, 483-95.

[79] Cole M A, Voelcker N H, Thiessen H, Griesser, H J, 2009 Biomaterials 30 (9) 1827-1850

[80] Geissler A, Vallat M F, Fioux P, Thomann J S, Frisch B, Voegel J C, Hemmerlé J, Schaaf P and Roucoules V, 2009, Plasma Process. and Polym., 7 (1), 64-77 